\title{
Normalization of aberrant pretherapeutic dynamic functional connectivity of extrastriate visual system in patients who underwent thalamotomy with stereotactic radiosurgery for essential tremor: a resting-state functional MRI study
}

\author{
${ }^{*}$ Constantin Tuleasca, MD, PhD, ${ }^{1-6}$ Thomas A. W. Bolton, MSc, ${ }^{7,8}$ Jean Régis, MD, ${ }^{9}$ \\ Elena Najdenovska, PhD, ${ }^{4}$ Tatiana Witjas, MD, ${ }^{10}$ Nadine Girard, MD, ${ }^{11}$ Francois Delaire, MSc, ${ }^{9}$ \\ Marion Vincent, MSc, ${ }^{9}$ Mohamed Faouzi, PhD, ${ }^{12}$ Jean-Philippe Thiran, PhD, ${ }^{5,13}$ \\ Meritxell Bach Cuadra, PhD, ${ }^{4,5}$ Marc Levivier, MD, PhD, ${ }^{3,6}$ and Dimitri Van De Ville, $\mathrm{PhD}^{7,8}$
}

\begin{abstract}
${ }^{1}$ Service de Neurochirurgie, Assistance Publique-Hôpitaux de Paris, Hôpitaux Universitaires Paris-Sud, Centre Hospitalier Universitaire de Bicêtre, Paris; 'Faculté de Médecine, Sorbonne Université, Paris, France; ${ }^{3}$ Neurosurgery Service and Gamma Knife Center, Centre Hospitalier Universitaire Vaudois, Lausanne; ${ }^{4}$ Medical Image Analysis Laboratory and Department of Radiology-Center of Biomedical Imaging, Centre Hospitalier Universitaire Vaudois, Lausanne; ${ }^{5}$ Signal Processing Laboratory (LTS 5), École Polytechnique Fédérale de Lausanne; ${ }^{6}$ Faculty of Biology and Medicine University of Lausanne; ${ }^{7}$ Medical Image Processing Laboratory, École Polytechnique Fédérale de Lausanne, Switzerland; ${ }^{8}$ Department of Radiology and Medical Informatics, University of Geneva, Switzerland; ${ }^{9}$ Stereotactic and Functional Neurosurgery Service and Gamma Knife Unit, CHU Timone, Marseille; ${ }^{10}$ Neurology Department, CHU Timone, Marseille; ${ }^{11}$ Department of Diagnostic and Interventional Neuroradiology, Centre de Résonance Magnétique Biologique et Médicale, Unité Mixte de Recherche, Centre National de la Recherche Scientifique, Faculté de Médecine et Assistance Publique-Hôpitaux de Marseille, Hôpital Timone, Marseille, France; ${ }^{12}$ Institute of Social and Preventive Medicine, Lausanne; and ${ }^{13}$ Department of Radiology, Centre Hospitalier Universitaire Vaudois, Lausanne, Switzerland
\end{abstract}

\begin{abstract}
OBJECTIVE The tremor circuitry has commonly been hypothesized to be driven by one or multiple pacemakers within the cerebello-thalamo-cortical pathway, including the cerebellum, contralateral motor thalamus, and primary motor cortex. However, previous studies, using multiple methodologies, have advocated that tremor could be influenced by changes within the right extrastriate cortex, at both the structural and functional level. The purpose of this work was to evaluate the role of the extrastriate cortex in tremor generation and further arrest after left unilateral stereotactic radiosurgery thalamotomy (SRS-T).

METHODS The authors considered 12 healthy controls (HCs, group 1); 15 patients with essential tremor (ET, rightsided, drug-resistant; group 2) before left unilateral SRS-T; and the same 15 patients (group 3 ) 1 year after the intervention, to account for delayed effects. Blood oxygenation level-dependent functional MRI during resting state was used to characterize the dynamic interactions of the right extrastriate cortex, comparing $\mathrm{HC}$ subjects against patients with ET before and 1 year after SRS-T. In particular, the authors applied coactivation pattern analysis to extract recurring wholebrain spatial patterns of brain activity over time.

RESULTS The authors found 3 different sets of coactivating regions within the right extrastriate cortex in $\mathrm{HCs}$ and patients with pretherapeutic ET, reminiscent of the "cerebello-visuo-motor," "thalamo-visuo-motor" (including the targeted thalamus), and "basal ganglia and extrastriate" networks. The occurrence of the first pattern was decreased in pretherapeutic ET compared to HCs, whereas the other two patterns showed increased occurrences. This suggests a misbalance between the more prominent cerebellar circuitry and the thalamo-visuo-motor and basal ganglia networks. Multiple
\end{abstract}

\footnotetext{
ABBREVIATIONS $\mathrm{ADL}=$ activities of daily living; $\mathrm{BA}=$ Brodmann area; $\mathrm{BOLD}=$ blood oxygenation level-dependent; $\mathrm{CAP}=$ coactivation pattern; $\mathrm{CHU}=\mathrm{Centre}$ Hospitalier Universitaire; $\mathrm{dFC}=$ dynamic functional connectivity; $\mathrm{DMN}$ = default mode network; $\mathrm{DTI}=$ diffusion tensor imaging; $\mathrm{ET}$ = essential tremor; fMRI = functional MRI; GMD = gray matter density; HC = healthy control; PCC = posterior cingulate cortex; QUEST = Quality of Life in Essential Tremor; ROI = region of interest; SRS-T = stereotactic radiosurgery thalamotomy; TSTH = tremor score on the treated hand; Vim = ventral intermediate nucleus. SUBMITTED December 11, 2018. ACCEPTED February 12, 2019. INCLUDE WHEN CITING Published online May 10, 2019; DOI: 10.3171/2019.2.JNS183454.

${ }^{*}$ C.T. and T.B. contributed equally to this work and share first authorship.
} 


\begin{abstract}
regression analysis showed that pretherapeutic standard tremor scores negatively correlated with the increased occurrence of the thalamo-visuo-motor network, suggesting a compensatory pathophysiological trait. Clinical improvement after SRS-T was related to changes in occurrences of the basal ganglia and extrastriate cortex circuitry, which returned to $\mathrm{HC}$ values after the intervention, suggesting that the dynamics of the extrastriate cortex had a role in tremor generation and further arrest after the intervention.
\end{abstract}

CONCLUSIONS The data in this study point to a broader implication of the visual system in tremor generation, and not only through visual feedback, given its connections to the dorsal visual stream pathway and the cerebello-thalamocortical circuitry, with which its dynamic balance seems to be a crucial feature for reduced tremor. Furthermore, SRS-T seems to bring abnormal pretherapeutic connectivity of the extrastriate cortex to levels comparable to those of HC subjects.

https://thejns.org/doi/abs/10.3171/2019.2.JNS183454

KEYWORDS radiosurgery; thalamotomy; ventral intermediate nucleus; extrastriate; visual; resting state; functional magnetic resonance imaging; coactivation pattern analysis; functional neurosurgery

$\mathrm{E}$ SSENTIAL tremor (ET) is currently considered the most common movement disorder in older people, affecting approximately $4 \%$ of the population above the age of 65 years. ${ }^{28}$ The cardinal symptom is gradual tremor appearance, which is principally in the upper limbs, although other parts of the body can be involved, including head, voice, legs, or trunk. ${ }^{12}$ The pathophysiology of ET remains unknown, although several mechanisms have been proposed.

One theory commonly presumes that tremor is generated by activity in the cerebello-thalamo-cortical network, including the ipsilateral motor cortex, corresponding motor thalamic area, and contralateral cerebellum, as segments of what is termed the "tremor network."15 This tremor network hypothesis has been supported by both human electrophysiology ${ }^{19}$ and neuroimaging studies. ${ }^{15}$ Furthermore, it is known that ventral intermediate nucleus (Vim) targeting generates good therapeutic responses, either by standard techniques, such as deep brain stimulation $^{4}$ or radiofrequency thermocoagulation,,$^{18}$ or by minimally invasive stereotactic radiosurgery (SRS) ${ }^{10,24}$ and high-intensity focused ultrasound. ${ }^{13,25}$ In a second theory, the inferior olivary nucleus is hypothesized to induce, supposedly by reticulospinal and vestibulospinal pathways, a rhythmic oscillatory activity that has been reported in several animal experiments. ${ }^{27} \mathrm{~A}$ third theory is related to a progressive cell loss in the background of a neurodegenerative disorder or a localized $\gamma$-aminobutyric acidergic dysfunction. ${ }^{34}$ More recently, the involvement of a widespread visually sensitive network in ET has been evoked, ${ }^{1,37,41}$ and would involve both the structural and functional levels. ${ }^{40}$

It is important to understand how the tremor circuitry interacts with other networks in order to ensure optimum functional brain homeostasis. Understanding whether visual feedback is important in tremor initiation and/or further tremor arrest after interventional techniques currently performed in clinical settings would enable us to broaden our understanding and description of ET pathways, with further potential therapeutic implications. ${ }^{39}$ It has recently been acknowledged that the blood oxygenation level-dependent (BOLD) signal in the primary visual cortex positively correlates with tremor severity. ${ }^{30}$ Moreover, other authors have advocated that vision is of major importance for accomplishing basic motor endeavors, particularly those requiring a degree of fine motor control and dexterity. ${ }^{20}$

Recently, we suggested that a cerebello-thalamo-visuo-motor network is responsible for tremor arrest after thalamotomy performed by SRS. ${ }^{38} \mathrm{We}$ advocated that this pathophysiological trait of visual system association with tremor is explanatory for a more prominent capacity, rather than an individual adaptive feature. We suggested that interconnectivity between the cerebellum lobule VI and bilateral motor cortex would play a pivotal role in sensory guidance of movements of the hands and fingers, and furthermore in movement control, ${ }^{37}$ positioning the cerebellum as a key player between the visual and motor systems. We thus hypothesized that the input from the visual to the contralateral motor network could be regulated by the cerebellum, which was also based on previously published data ${ }^{31}$ Furthermore, other authors have suggested a similar circuitry, based on task-based functional MRI (fMRI) findings. ${ }^{4}$ More recently, Benito-León et al. ${ }^{5}$ confirmed some of our group's findings and suggested a role of the right lingual gyrus, among other anatomical structures, in relation to the control of movement sequencing, and therefore having relevance in patients with tremor. Based on this hypothesis we sought to make a step forward, in an attempt to better understand the role of the visual system as well as its potential association with tremor generation and further arrest after interventional procedures such as thalamotomy.

The present study considers 3 different types of subjects for subsequent resting-state fMRI analysis: healthy controls (HCs), and ET patients before and 1 year after left unilateral Vim SRS thalamotomy (SRS-T). Nowadays, it is widely known that the brain is active even in the absence of overt behavior. ${ }^{7}$ Furthermore, spatial patterns of spontaneous activity might involve different subregions of a network at different moments in time, possibly reflecting functionally relevant relationships that cannot be captured by conventional correlational analyses and that have raised interest in dynamic functional connectivity (dFC). ${ }^{31}$ Therefore, we use a recent methodology, termed coactivation pattern (CAP) analysis, which allows us to investigate how a particular seed region interacts with the rest of the brain in a time-varying manner. ${ }^{26}$ To investigate this, we examined a subpart of the right extrastriate cortex 
(Brodmann area [BA] 19, including V3, V4, and V5) as a unique region of interest (ROI). It was chosen from our previously published data ${ }^{37,40}$ (further confirmed by taskbased studies ${ }^{1}$ ), given its functional connectivity with the cerebellum lobule VI, bilateral motor cortex, and frontal eye fields in ET. We determined the different whole-brain network patterns (i.e., CAPs) that occur over time when this ROI is active. We then extracted the occurrences of each CAP and correlated them with tremor severity, using clinically relevant scores. We hypothesized, based on previous findings, $1,35,38,40$ that the extrastriate cortex in ET presents adaptive changes, in synchrony with the cerebello-thalamo-cortical axis, as a part of a multicomponent oscillatory system. Finally, we examined whether this oscillatory system responds to interventional procedures, such as Vim SRS-T, and further correlated the BOLD response signal not only 1 year after such intervention, but also in the $\mathrm{HC}$ group.

\section{Methods}

\section{Study Subjects}

The study included 42 neuroimaging sessions performed in $12 \mathrm{HC}$ subjects (group 1), and 15 consecutive cases with ET in the pretherapeutic (group 2; right-sided, drug-resistant ET) or posttherapeutic (group 3) state (i.e., a total of 30 resting-state fMRI recordings). Formal approval was acquired (Centre Hospitalier Universitaire [CHU] Timone Ethical Committee), with further individual written informed consent. The patients were referred by T.W., a movement disorders specialized neurologist at $\mathrm{CHU}$ Timone, Marseille, France. Thalamotomy was performed using SRS between September 2014 and April 2016. All individuals who underwent SRS-T were consecutive patients who fulfilled the inclusion criteria, with clear diagnosis of ET (patients with Parkinson disease, mixed tremor, or other such conditions were excluded); no other structural abnormalities (e.g., hippocampal sclerosis); and rigorous Power motion index analysis (see below) to avoid spurious correlations (refer to Supplemental Text, Power motion index cite). Data were analyzed independently by 3 people in Paris and in Lausanne (C.T., T.B., and D.V.D.). These people were not involved in the patients' selection, SRS-T treatment, or follow-up course. The mean age in the HC group was 69.4 years (range $60-83$ years) and the male/female ratio was 5:7. For the ET group, the mean age at treatment was 70 years (range 51-79 years), the mean duration of symptoms at treatment was 35.1 years (range 6-62 years), and the male/female ratio was 3:12.

The neurological evaluation was always performed by T.W. The neuroimaging evaluation was done on a single 3-T MR machine (Siemens Skyra); T1-weighted and resting-state fMRI $(n=15)$ were performed. Because the medication in this cohort was frequently ineffective, most of the patients no longer received drug treatment at the time of SRS-T. Nevertheless, T.W. performed the clinical assessment while the patient was receiving pharmacological therapy (as was the case in only a minority of situations ${ }^{45}$ ), although the scanning procedure itself was performed in a drug-naïve state (with drugs being stopped at least 3 days prior to scanning, for both the pre- and post-
TABLE 1. Clinical data

\begin{tabular}{|c|c|c|c|c|}
\hline Variable & Mean & SD & Minimum & Maximum \\
\hline ADL baseline score & 28 & 11.6 & 13 & 49 \\
\hline $\begin{array}{l}\text { ADL score } 1 \text { yr after SRS } \\
\text { (drop in points) }\end{array}$ & -23.6 & 12.7 & 2 & -48 \\
\hline TSTH at baseline & 19 & 5.8 & 8 & 30 \\
\hline $\begin{array}{l}\text { TSTH } 1 \text { yr after SRS (drop } \\
\text { in points) }\end{array}$ & -13 & 5.2 & -4 & -22 \\
\hline Head tremor baseline score & 1.06 & 0.9 & 0 & 2 \\
\hline $\begin{array}{l}\text { Head tremor score } 1 \text { yr after } \\
\text { SRS (drop in points) }\end{array}$ & -0.6 & 1.07 & 1 & -2 \\
\hline QUEST baseline score & 45.8 & 16 & 29 & 80 \\
\hline $\begin{array}{l}\text { QUEST score } 1 \text { yr after } \\
\text { SRS (drop in points) }\end{array}$ & -22 & 14.8 & -2 & -44 \\
\hline $\begin{array}{l}\text { Time to tremor arrest after } \\
\text { thalamotomy (days) }\end{array}$ & 133 & 89.1 & 15 & 300 \\
\hline
\end{tabular}

therapeutic states). The same neuroimaging protocol was repeated 1 year after SRS-T $(n=15)$.

\section{Clinical Evaluation and Outcome Measures}

Clinical evaluation was made using the following instruments: standard tremor scores determined every 3 months after thalamotomy by the survey designed by Bain et al. ${ }^{3}$ (activities of daily living [ADL]); the tremor score on the treated hand (TSTH, right hand) from the Fahn-TolosaMarin tremor rating scale; ${ }^{14}$ head tremor $(n=15)$, evaluated using the Tremor Research Group Essential Tremor Rating Assessment (0-3); or Quality of Life in Essential Tremor (QUEST) score. ${ }^{36}$ For clinical data, refer to Table 1.

\section{SRS-T Procedure}

All SRS-T procedures were performed in Marseille (CHU Timone) by J.R., who used the Leksell Gamma Knife and the associated Leksell GammaPlan software (Elekta Instruments AB). To avoid artifacts we first acquire, pretherapeutically, diffusion tensor imaging (DTI) data without the frame. We further coregister the DTI study with the therapeutic stereotactic images. We always apply the Leksell coordinate G Frame (Elekta AB) under local anesthesia the day of the thalamotomy. After the frame is placed, patients undergo both stereotactic $\mathrm{CT}$ and MRI. We further define the landmarks of interest, including the anterior and posterior commissures, which were identified on the MR ventriculography study (T2 CISS/FIESTA sequence, Siemens). We perform uniform indirect targeting using the Guiot diagram, ${ }^{37}$ placed $2.5 \mathrm{~mm}$ above the anterior commissure-posterior commissure line and $11 \mathrm{~mm}$ lateral to the wall of the third ventricle. We always use a single 4-mm isocenter. A maximum prescription dose of 130 Gy at the $100 \%$ isodose line is uniformly prescribed. ${ }^{37}$

\section{Coactivation Patterns}

The MRI data acquisition and preprocessing details can be found in the Supplemental Text. 
A whole group of 27 participants (see Study Subjects section; $\mathrm{n}=12 \mathrm{HC}$ subjects plus $\mathrm{n}=15$ patients with pretherapeutic ET who underwent scanning before and 1 year after SRS-T) was used for the analysis. We examined the time courses of a subpart of the right extrastriate cortex (BA 19, including V3, V4, and V5) as a unique ROI. This ROI was chosen from our previously published data, 37,40 given its interconnection with the cerebellum lobule VI, bilateral motor cortex, and frontal eye fields in patients with ET. ${ }^{40}$ We evaluated the synchronous oscillations of this ROI by disentangling the set of whole-brain networks with which it activates over time. Furthermore, we correlated the occurrence of each network with tremor severity, using clinically relevant scores.

CAPs were generated using an in-house toolbox, created as part of our interest in $\mathrm{dFC} .{ }^{31} \mathrm{CAP}$ analysis is a frame-wise dFC tool that allows the identification of spatially distinct networks coactivating with a chosen ROI at given time points. They can then be further analyzed in terms of spatial and temporal features. The whole-brain CAPs with the right extrastriate cortex seed were initially computed for a group of 27 subjects $(12 \mathrm{HCs}$ and 15 patients with ET) by retaining fMRI volumes for which the seed signal exceeded a $\mathrm{z}$-scored value of $\mathrm{T}_{\text {seed }}=0.5$ and subsequently separating them through K-means clustering (spatial correlation used as distance measure, 100 iterations, 20 repetitions, random data points for initialization; see Supplemental Fig. 1). Similarly to previous CAP studies, ${ }^{26}$ only the $15 \%$ most active and $5 \%$ most deactive voxels in each fMRI volume were considered for the clustering step, also discarding all remaining nonnull clusters with fewer than 6 neighboring elements. A CAP was defined as the average of all frames attributed to a cluster.

For each CAP, we computed a dynamically informative subject-specific metric: the occurrence number (i.e., the number of times entering the assessed state). Thus, larger occurrence values reflect a more frequent expression of a given CAP during moments of seed activity. The retained fMRI volumes from posttherapeutic ET sessions, which were not used in the generation of the CAPs, were assigned to their closest respective CAP as quantified through spatial correlation, if the similarity exceeded the 5 th percentile of the similarity distribution of the frames constituting the assessed CAP.

Here, we report the results obtained with $\mathrm{K}=3$ CAPs. Refer to Supplemental Fig. 1 and the associated legend for the justification of this choice.

\section{Statistical Analysis}

One-way multivariate ANOVA with repeated measures (STATA version 11; StataCorp LLC) was considered appropriate, because there were no outliers seen by boxplot and Mahalanobis distance ( $\mathrm{p}>0.001$ ), no multicollinearity $(r<0.65)$, and homogeneity of variance-covariance matrices (Box's M test, $p>0.001$ ).

The normalized occurrence numbers that significantly differed between groups were correlated with the clinical tremor scores and time to tremor arrest (see the Clinical Evaluation and Outcome Measures and Coactivation Patterns sections above). A p value $<0.05$ (Spearman's rank correlation coefficient) was considered statistically
TABLE 2. CAP characteristics

\begin{tabular}{cccc}
\hline Characteristic & $\mathrm{HCs}$ & $\begin{array}{c}\text { ET Before } \\
\text { Thalamotomy }\end{array}$ & $\begin{array}{c}\text { ET 1 Yr After } \\
\text { Thalamotomy }\end{array}$ \\
\hline Occurrence* $^{*}$ & & & \\
\hline $\mathrm{CAP}_{1}$ & $40(38,15-78)$ & $23.5(19,8-67)$ & $35.1(32,15-62)$ \\
\hline $\mathrm{CAP}_{2}$ & $16.6(17,4-43)$ & $26.1(25,10-44)$ & $16.4(19,2-26)$ \\
\hline $\mathrm{CAP}_{3}$ & $14.3(13,4-24)$ & $25.5(23,10-42)$ & $15.1(13,3-30)$ \\
\hline
\end{tabular}

Values are expressed as the mean number of occurrences (median, range).

${ }^{*} p<0.001$ according to multivariate ANOVA.

significant. Data are presented as the median \pm SEM, unless otherwise stated.

\section{MR Signature Volume After SRS-T}

The MR signature volume after SRS-T has always been contoured on the T1-weighted, Gd-injected MR study obtained 1 year after the procedure, which is considered the final radiological aspect in our experience, ${ }^{44}$ as further confirmed by other groups. ${ }^{10}$ To guarantee the accuracy of this volume calculation, the individual patient's 1-year MR image was imported in the Leksell GammaPlan software (Elekta Instruments $\mathrm{AB}$ ) and coregistered with the posttherapeutic MR sequence. A manual drawing was made for each individual case. The mean thalamotomy volume after SRS-T was $0.140 \mathrm{ml}$ (median $0.072 \mathrm{ml}$, range 0.014$0.600 \mathrm{ml}$ ). There was a correlation of lesion volume with TSTH improvement $(\mathrm{p}=0.05)$, but not with a drop in ADL $(\mathrm{p}=0.19)$ or QUEST $(\mathrm{p}=0.29)$ score.

In the present research trial, one patient had a much larger 1-year MR signature, which was clinically silent. Moreover, the patient showed clinical alleviation of tremor. This case is not included in the present analyses, due to the rigorous inclusion criteria adopted for resting-state data with regard to the Power motion index.

\section{Cognitive Assessment}

The mean pretherapeutic Mattis Dementia Rating Scale $^{32}$ was 135.9 , and the posttherapeutic value was 135.5 $(\mathrm{p}>0.05)$.

\section{Results \\ Coactivation Patterns}

The retained frames (described in detail below and in Table 2) were attributed to the CAPs, which we categorized as follows: cerebello-visuo-motor $\left(\mathrm{CAP}_{1}: 38.6 \%\right)$; thalamo-visuo-motor $\left(\mathrm{CAP}_{2}: 32.6 \%\right)$; and basal ganglia and extrastriate cortex $\left(\mathrm{CAP}_{3}: 28.8 \%\right)$. All of the foregoing values represent the percentage of occurrence for the initial group, containing the $\mathrm{HC}$ subjects and patients with pretherapeutic ET.

\section{CAP : Cerebello-Visuo-Motor}

This CAP illustrates the cerebellum lobules VI and VIIIa bilaterally (Fig. 1 left). This is completed with the fusiform gyrus, visual areas V1, V2, V3, and V5, and the primary sensory-motor cortex. 


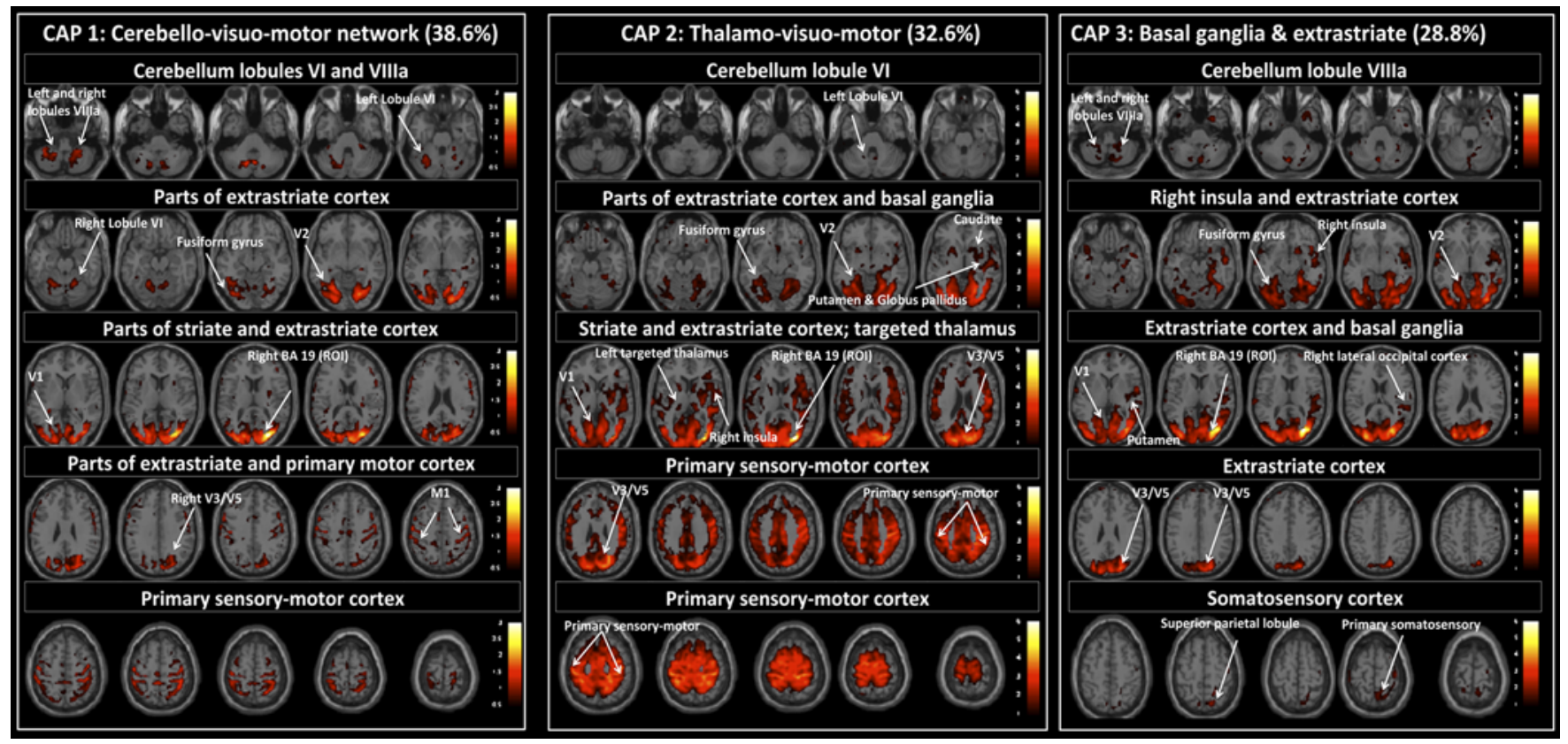

FIG. 1. Illustration of the 3 CAPs, from 1 to 3 (left to right). Figure is available in color online only.

A boxplot representation of occurrences (Fig. 2 left) revealed a decreased metric for the pretherapeutic ET (median 19, range 8-67) compared with the HC (median 38, range $15-78 ; p=0.02$ ) group. Occurrences increased to values similar to those in the HC group at 1 year after thalamotomy (median 32, range 15-62; $\mathrm{p}=0.47$ for $\mathrm{HC}$ and posttherapeutic ET comparison; $p=0.03$ for prethera- peutic and posttherapeutic ET comparison; $\mathrm{p}<0.001$ according to ANOVA).

$\mathrm{CAP}_{2}$ : Thalamo-Visuo-Motor, Including Targeted Thalamus

This CAP displays the left cerebellum lobule VI; fusiform gyrus; V1, V2, V3, and V5 areas; and the bilateral primary sensory-motor cortex (Fig. 1 center). Moreover,

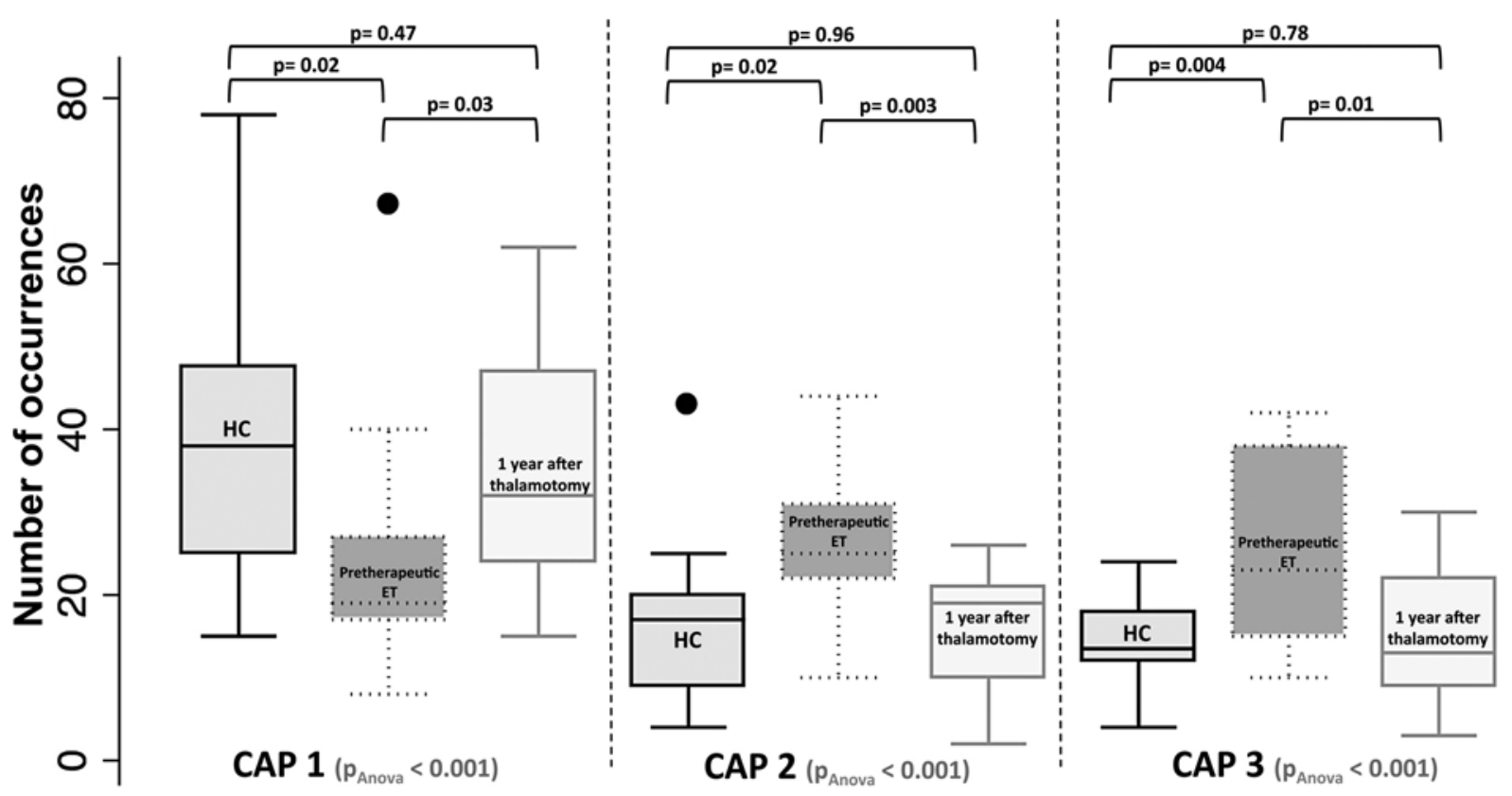

FIG. 2. Illustration of the number of occurrences, as boxplots, for each CAP in ET, HC, and post-SRS-T states, with associated $p$ values. Black circles in CAP1 and CAP2 boxplots represent outliers. 


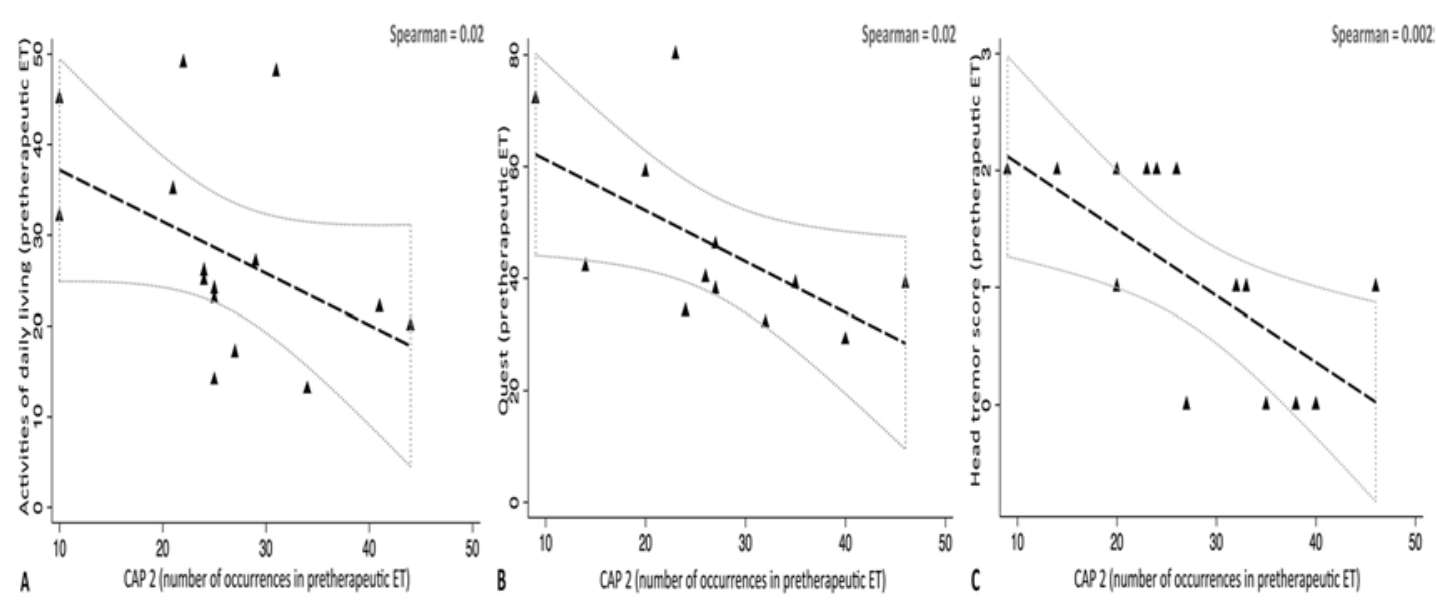

FIG. 3. Graphs showing correlation between pretherapeutic clinical scores and the number of occurrences for $\mathrm{CAP}_{2}(\mathrm{ADL}[\mathrm{A}]$, QUEST [B], and head tremor [C]).

one can visualize the left targeted thalamus as well as parts of the basal ganglia circuitry such as the caudate, putamen, and globus pallidus.

Inversely to the $\mathrm{CAP}_{1}$ case, the occurrences (Fig. 2 center) were increased in the pretherapeutic ET group (median 25, range 10-44) compared to the HC group (median 17 , range $4-43 ; p=0.02$ ), and further decreased to values similar to those in the HC group at 1 year after thalamotomy (median 19, range 2-26; $\mathrm{p}=0.96$ for $\mathrm{HC}$ and posttherapeutic ET comparison; $\mathrm{p}=0.003$ for pretherapeutic and posttherapeutic ET comparison; $\mathrm{p}<0.001$ according to ANOVA).

\section{$\mathrm{CAP}_{3}$ : Basal Ganglia and Extrastriate Cortex}

This CAP includes the left and right cerebellum lobule VIIIa; parts of the extrastriate cortex (fusiform gyrus, left V2, right V3, V5); the bilateral primary visual cortex V1;

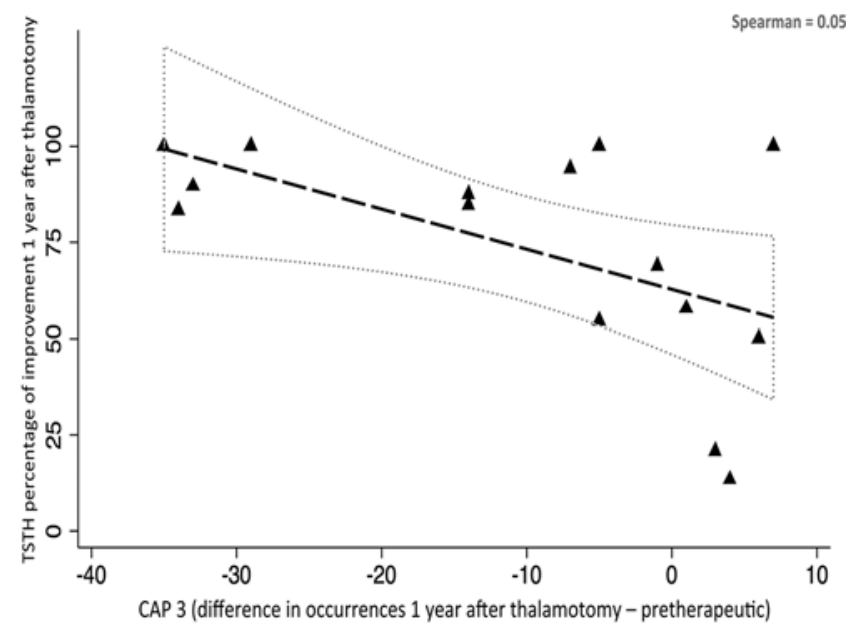

FIG. 4. Graphs showing correlation between posttherapeutic TSTH and difference in occurrences between the posttherapeutic and pretherapeutic states for $\mathrm{CAP}_{3}$. the right superior parietal lobule; and the right primary somatosensory cortex (Fig. 1 right). Furthermore, we visualize the right insula and parts of the basal ganglia such as the right putamen.

As for occurrences (Fig. 2 right), similarly to $\mathrm{CAP}_{2}$, there was an increase in the pretherapeutic ET group (median 23, range 10-42) compared to the $\mathrm{HC}$ group (median 13 , range 4-24; $p=0.004$ ), and further decrease to values similar to those in the HC group at 1 year after thalamotomy (median 13, range 3-30; $\mathrm{p}=0.78$ for $\mathrm{HC}$ and posttherapeutic ET comparison; $\mathrm{p}=0.01$ for pretherapeutic and posttherapeutic ET comparison; $\mathrm{p}<0.001$ according to ANOVA).

\section{Clinical Correlations}

We found statistically significant correlations between pretherapeutic tremor scores and pretherapeutic occurrence numbers for $\mathrm{CAP}_{2}$ (Fig. 3), and also between the difference in occurrences (1 year after SRS-T minus pretherapeutic) for $\mathrm{CAP}_{3}$ and the percentage of improvement in TSTH (Fig. 4).

\section{Correlations Between Pretherapeutic Tremor Scores and Number of Occurrences}

There were statistically significant negative correlations between pretherapeutic $\mathrm{CAP}_{2}$ (thalamo-visuo-motor) occurrence numbers and ADL (Fig. 3A), QUEST (Fig. 3B), and head tremor (Fig. 3C) scores, with Spearman's rank correlation coefficients of $0.02,0.02$, and 0.002 , respectively.

\section{Correlations Between Improvement in TSTH and Difference in Occurrence (1 Year After Thalamotomy Minus Pretherapeutic)}

There was a statistically significant negative correlation between the difference in occurrences for $\mathrm{CAP}_{3}$ (basal ganglia and extrastriate cortex) and the tremor score improvement (Spearman's rank correlation coefficient of 0.05; Fig. 4). 


\section{Analysis Performed as a Negative Control, Using Posterior Cingulate Cortex}

An analysis was performed as a negative control, using another seed not involved with tremor generation. The posterior cingulate cortex (PCC) was selected as the ROI in order to explore a classic resting-state network, the default mode network (DMN). The seed was extracted from the independent component analysis parcellation published by Shirer et al. (refer to Supplemental Text). The same number of clusters $(\mathrm{K}=3)$ and overall methodology were kept compared to the main results. Supplemental Fig. 2 depicts the respective CAPs (upper half) and their associated occurrences across conditions (lower half), presented as boxplots. Interestingly, $\mathrm{CAP}_{1}$ contains the bilateral thalamus, with the visual and sensory-motor networks also, whereas $\mathrm{CAP}_{2}$ represents the classic DMN, including the anterior prefrontal cortex, $\mathrm{PCC}$, bilateral angular gyrus, and lateral temporal cortex. $\mathrm{CAP}_{3}$ highlights the posterior DMN with the bilateral motor cortex and visual association cortex.

For this particular seed and the related counts, no statistically significant relationship was found.

\section{Discussion}

The present study evaluates resting-state $\mathrm{dFC}$ of the extrastriate cortex (BA 19-including V3, V4, and V5) at the whole-brain level, using CAP analysis. This analysis reveals a new perspective for identifying components of moment-to-moment brain activity and, subsequently, linking their temporal features to clinically functional properties, which is an important next step toward systems-level models. ${ }^{22}$ This provides new insights into the role of the extrastriate cortex and its dynamic changes in functional connectivity before and after SRS-T. Three relevant CAPs of the selected seed ROI are revealed: $\mathrm{CAP}_{1}$, cerebello-visuo-motor; $\mathrm{CAP}_{2}$, thalamo-visuo-motor; and $\mathrm{CAP}_{3}$, basal ganglia and extrastriate cortex. The relative occurrence percentages of these CAPs are similar and not statistically different ( $\mathrm{p}>0.05 ; \mathrm{CAP}_{1}, 38.6 \%$; $\mathrm{CAP}_{2}, 32.6 \%$; and $\mathrm{CAP}_{3}$, $28.8 \%$ ) in terms of occurrences for HCs and patients with pretherapeutic ET. Across conditions, $\mathrm{CAP}_{1}$ had decreased pretherapeutic occurrences compared with HCs, whereas it was the opposite for $\mathrm{CAP}_{2}$ and $\mathrm{CAP}_{3}$. For all CAPs, the number of occurrences after SRS-T came back to the HC level ( $\mathrm{p}>0.05)$.

$\mathrm{CAP}_{2}$ includes the left targeted thalamus, at the level of the Vim, which illustrates a functional circuitry that links the motor thalamus and the extrastriate cortex. $\mathrm{CAP}_{2}$ occurrences correlate, before thalamotomy, with pretherapeutic standard tremor scores. In fact, lower pretherapeutic occurrences (close to HCs) were associated with higher ADL, QUEST, and head tremor scores. In other words, higher occurrences correspond to lower pretherapeutic functional impairment, suggesting an adaptive trait in the patients with ET. In fact, $\mathrm{CAP}_{2}$ also contains the left cerebellum lobule VI, parts of the extrastriate cortex, the left Vim, and the primary sensory-motor cortex. We postulated the theory of a cerebello-thalamo-visuo-motor network in an earlier SRS-fMRI study, ${ }^{37,40}$ which was further confirmed by other authors ${ }^{1}$ and by the present find- ings. In addition, parts of the basal ganglia, including the caudate, putamen, and globus pallidus, were also included. The presence of these structures is not astonishing with respect to the already acknowledged pathophysiological aspects of ET.

In $\mathrm{CAP}_{3}$, the percentage of improvement in the TSTH (right hand) at 1 year after SRS-T correlates with the drop in occurrences after the intervention, suggesting that $\mathrm{CAP}_{3}$, which includes the basal ganglia and extrastriate cortex, together with the right superior parietal lobule and primary sensory-motor cortex, is part of a compensatory mechanism in pretherapeutic ET, further responding to SRS-T. $\mathrm{CAP}_{3}$ also particularly includes the dorsal visual stream, which is commonly considered a vision-for-action pathway. The dorsal visual stream is mainly linked with visually guided reaching and grasping of objects, based on several aspects, such as the moment-to-moment analysis of their spatial location, orientation, and shape. Furthermore, the dorsal stream also overviews tools as a group, so that easily manageable objects would be processed by those brain regions that are important for the execution of actions.

\section{Tracing the Role of a Visually Sensitive Network in ET}

There is an emerging body of literature with regard to the potential role of the visual system in tremor, as well as of a visually sensitive structural and functional network in tremor in general, and in ET in particular. It has been previously advocated that vision is of major importance for the performance of basic motor tasks, particularly tasks necessitating a grade of fine motor control and dexterity. ${ }^{11}$ Furthermore, reports of the tremor-vision association have provided subjects with an amplified view of their tremor. ${ }^{43}$ The hypothesis was that, in neurologically intact subjects, a potential tremor presence at the level of the distal part is so small that sensitive variations in tremor amplitude cannot be noticed by the visual system. ${ }^{33}$ One might think that by increasing the tremor image (but not tremor itself), the visual system might be better able to perceive the smallamplitude tremor inherent to each limb segment. Furthermore, this type of reaction could potentially allow for greater control of the limb segment in question. This has not been conclusively demonstrated, in the sense that augmented visual feedback would minimize finger tremor. ${ }^{43}$ Most probably, although visual feedback plays a role, other factors might be involved in tremor influence, such as the goal of the task, the number of the involved segments, the dexterity, and so on.

Using a one-sided pointing assignment under circumstances in which the visual feedback, limb used, and target size were transformed, Keogh et al. ${ }^{23}$ have investigated physiological tremor present in the upper limb of 8 adults. Postural tremor from the parts of the upper extremity, forearm muscle EMG activity, and target accuracy measures were documented and examined in the time and frequency fields. The authors established that physiological tremor generation observed in neurologically intact subjects is not just the creation of intrinsic oscillations, but is task dependent and can be influenced by changes in the information provided to the subject. Altering the kind of visual feedback caused increased tremor amplitude at the 
index finger. Furthermore, varying the nature of the visual feedback influences tremor.

Additional evidence came from structural studies evaluating pretherapeutic gray matter density (GMD), but also its changes after thalamotomy for ET, as related to clinical effect. We have recently published two voxel-based morphometry reports, ${ }^{41,42}$ in which we postulate that a widespread visually sensitive structural network in ET is affected by thalamotomy. In the first report ${ }^{41}$ we evaluated GMD changes between the pre- and posttherapeutic states following SRS-T. We reported as statistically significant GMD alterations within the left temporal pole (BA 38) and, more importantly, a larger cluster including the left occipital lobe (BA 19, V4, V5, and parahippocampal place area). In the second voxel-based morphometry study, ${ }^{42}$ we showed that pretherapeutic GMD in the right extrastriate cortex (BA 18) predicted TSTH improvement 1 year after thalamotomy (which is classically considered a crucial point for evaluating clinical and radiological changes after SRS-T). Additional studies performed using DTI ${ }^{6}$ have revealed visuospatial function correlation with the right parietooccipital lobe by using white matter integrity measures derived from DTI in patients with ET.

Furthermore, functional studies have brought new evidence of a visually sensitive network in ET. In an observational study, Archer et al. ${ }^{1}$ used task-based fMRI and suggested the involvement of the extrastriate areas V3 and V5, as related to tremor severity in patients with ET. Their conclusion was that the severity of tremor is exacerbated by increased visual feedback, suggesting that designers of new computing technologies should consider using lower visual feedback to reduce tremor in patients with ET. This aspect is in perfect concordance with previous observations in a randomized trial conducted in patients with ET, which concluded that tremor significantly improved in the absence of a visual feedback..$^{16}$

In an earlier work we implemented an interventional study and investigated patients with ET by using restingstate fMRI data ${ }^{37}$ acquired pre- and postherapeutically (at 1 year after SRS-T). We used data-driven multivariate analysis (i.e., independent component analysis ${ }^{9}$ ) to conduct whole-brain analyses without a prior hypothesis in the computational approach and/or the statistical model. We reported on two networks ${ }^{40}$ that had statistically noteworthy interconnectivity with visual areas. The first network revealed the interaction between time (pre- and 1 year posttherapeutic) and clinical effects; this encompassed the bilateral motor network, frontal eye fields, and left cerebellum lobule VI, in which network interconnectivity strength with right visual BA 19 (which is actually the investigated ROI in the present study) related to tremor arrest after SRS-T. ${ }^{37}$ The second network revealed the time effect independently of the clinical one; this included part of the salience network, which showed altered interconnectivity with the right fusiform gyrus and visual association area V5. We further suggested that SRS-T seems to restore interconnectivity in the visual areas to normal for all patients, but those patients in whom this region was more functionally integrated pretherapeutically had much larger clinical benefit. One recent resting-state FDG-PET/ CT study considered the brain glucose metabolism in 10 patients with ET, ${ }^{20}$ and the authors also reported an unexpected hypermetabolism in primary and association visual cortices.

\section{The Cerebellum: A Key Player Between the Visual System and Tremor Axis}

It is now well understood that the cerebello-thalamocortical loops play a major role in the emergence of pathological tremor and voluntary rhythmic movements. ${ }^{29} \mathrm{Re}-$ cent evidence has even suggested that they differ and have specific fingerprints between essential, parkinsonian, and mimicked tremors. ${ }^{29}$ However, notwithstanding the subtle variances in cerebellar source topography, the authors suggested no evidence that the cerebellum is the source of oscillation in ET, or that the cortico-bulbo-cerebello-thalamo-cortical loop displays diverse tremorogenic roles. ${ }^{29}$ Other studies have revealed, by coherent source activity, parts of the "tremor axis," including the cerebellum, the thalamus, or the motor and premotor cortex. ${ }^{35}$ Moreover, some authors considered the cerebellar source in ET to be the motor cerebellum. ${ }^{8}$ Recently, Joutsa et al. ${ }^{21}$ used a technique called lesion network mapping and proposed that in patients with heterogeneous lesion location involved in tremor relief the networks were all connected to common nodes in the cerebellum and the thalamus.

The present study displays parts of the cerebellum lobule VI within the first two CAPs, suggesting a calibration by this anatomical area of the relationship between the cerebello-thalamo-cortical axis and the visual system. This is reflected in the anatomical clusters that include the motor cerebellum, the left motor targeted thalamus, parts of the right extrastriate cortex, and bilateral M1. Recently, we postulated that a cerebello-thalamo-visuo-motor network is responsible for tremor arrest after SRS-T. ${ }^{40}$ We advocated that this pathophysiological feature is explanatory for a more prominent role, rather than constituting a simple, individual, adaptive trait. We suggested that interconnectivity between the cerebellum lobule VI and bilateral motor cortex would play a pivotal role in sensory guidance of movements of the hands and fingers, and furthermore in movement control, placing the cerebellum as a key player between the visual and motor systems. We thus hypothesized that the input from the visual to the contralateral motor network could be regulated by the cerebellum, which was also based on previously published data. ${ }^{17}$ Furthermore, other authors have suggested a similar circuitry in light of task-based fMRI findings. ${ }^{2}$

Despite our particular care, this study has several inherent limitations. The first one is the small number of subjects, which was mainly related to the fact that we wanted to extract from our cohort only patients with ET who had been consecutively treated, therefore benefiting from uniform clinical and radiological follow-up. The second is related to the sensitivity of CAP analysis to even small amounts of movement: although the initial cohort was larger, to minimize the possibility of spurious contributions to the results, we used a very conservative Power motion index threshold. The third limitation is related to the design of the study and the use of resting-state data: some authors might argue that the resting state is not necessarily directly related to motor performance in general 
and to that of the individual in particular; however, we aimed to evaluate network changes in the absence of any task. The fourth limitation is the absence of a control set on longitudinal changes (at 1 year) in an $\mathrm{HC}$ group similar to the pretherapeutic one; nevertheless, recent studies have advocated the reproducibility of functional networks across multiple sessions, including at 1-year intervals. ${ }^{24} \mathrm{~A}$ fifth limitation is that the neurological evaluation was not blinded-it was performed by the same neurologist (T.W.) who evaluated patients pre- and posttherapeutically. A sixth limitation is that the exact time point when BOLD changes correlate with clinical improvement remains unknown.

\section{Conclusions}

We found that resting-state $\mathrm{dFC}$ of the extrastriate cortex can be characterized by 3 CAPs. These patterns corresponded to 1) a "cerebello-visuo-motor," 2) a "thalamovisuo-motor," and 3) a "basal ganglia and extrastriate" network. The first one has fewer occurrences in pretherapeutic patients with ET compared to HCs, whereas the other two patterns display increased occurrences. This would suggest a balance between the cerebellar circuitry and the thalamo-visuo-motor and also the basal ganglia ones. The pretherapeutic tremor scores correlated with the abnormal increase in occurrences of the thalamo-visuo-motor network, suggesting a compensatory pathophysiological trait. The improvement in tremor scores after SRS-T is more related to changes within the basal ganglia and extrastriate cortex. All these circuitries aligned to values in $\mathrm{HC}$ subjects after SRS-T. Although the present data could be seen as purely associative, this might, however, suggest a prominent role of the extrastriate cortex and its dynamic connectivity in tremor generation and further arrest after interventional procedures. Because the negative seed control by the classic resting-state PCC area showed no relationship with the clinical data, this also further strengthens our belief that thalamotomy performed using SRS normalized aberrant pretherapeutic functional connectivity of the visual association cortex, in line with patients' clinical improvement after the intervention.

This opens the discussion for a potential new target for tremor, as we have previously advocated in earlier studies, aiming at the extrastriate cortex..$^{37,40-42}$ These findings support the idea that the visual system might play a prominent role in tremor generation and further arrest after interventional procedures such as SRS-T. However, such a hypothesis should be validated in larger cohorts, using multimodal approaches.

\section{Acknowledgments}

This work was supported by the Swiss National Science Foundation (SNSF-205321-157040) and by the Centre d'Imagerie BioMédicale of the University of Lausanne; the École Polytechnique Fédérale de Lausanne; the University of Geneva; the Centre Hospitalier Universitaire Vaudois; the CHU Timone, Marseille, France; the Hôpitaux Universitaires de Genève; and the Leenaards and Louis-Jeantet Foundations. We are grateful to the Lausanne and Marseille University Hospitals, and acknowledge the major contribution of Axelle Cretol for keeping the database of the patients up to date, and for all her administrative work.

\section{References}

1. Archer DB, Coombes SA, Chu WT, Chung JW, Burciu RG, Okun MS, et al: A widespread visually-sensitive functional network relates to symptoms in essential tremor. Brain 141:472-485, 2018

2. Archer DB, Coombes SA, Chu WT, Woo Chung J, Burciu RG, Okun MS, et al: Reply: Visually-sensitive networks in essential tremor: evidence from structural and functional imaging. Brain 141:e48, 2018

3. Bain PG, Findley LJ, Atchison P, Behari M, Vidailhet M, Gresty M, et al: Assessing tremor severity. J Neurol Neurosurg Psychiatry 56:868-873, 1993

4. Benabid AL, Pollak P, Gao D, Hoffmann D, Limousin P, Gay $\mathrm{E}$, et al: Chronic electrical stimulation of the ventralis intermedius nucleus of the thalamus as a treatment of movement disorders. J Neurosurg 84:203-214, 1996

5. Benito-León J, Serrano JI, Louis ED, Holobar A, Romero JP, Povalej-Bržan P, et al: Essential tremor severity and anatomical changes in brain areas controlling movement sequencing. Ann Clin Transl Neurol 6:83-97, 2019

6. Bhalsing KS, Kumar KJ, Saini J, Yadav R, Gupta AK, Pal PK: White matter correlates of cognitive impairment in essential tremor. AJNR Am J Neuroradiol 36:448-453, 2015

7. Biswal B, Yetkin FZ, Haughton VM, Hyde JS: Functional connectivity in the motor cortex of resting human brain using echo-planar MRI. Magn Reson Med 34:537-541, 1995

8. Broersma M, van der Stouwe AMM, Buijink AWG, de Jong BM, Groot PFC, Speelman JD, et al: Bilateral cerebellar activation in unilaterally challenged essential tremor. Neuroimage Clin 11:1-9, 2015

9. Calhoun VD, Adali T, Pearlson GD, Pekar JJ: A method for making group inferences from functional MRI data using independent component analysis. Hum Brain Mapp 14:140 151,2001

10. Campbell AM, Glover J, Chiang VL, Gerrard J, Yu JB: Gamma knife stereotactic radiosurgical thalamotomy for intractable tremor: a systematic review of the literature. Radiother Oncol 114:296-301, 2015

11. Cesari P, Newell KM: The scaling of human grip configurations. J Exp Psychol Hum Percept Perform 25:927-935, 1999

12. Elble RJ: Diagnostic criteria for essential tremor and differential diagnosis. Neurology 54 (11 Suppl 4):S2-S6, 2000

13. Elias WJ, Lipsman N, Ondo WG, Ghanouni P, Kim YG, Lee $\mathrm{W}$, et al: A randomized trial of focused ultrasound thalamotomy for essential tremor. N Engl J Med 375:730-739, 2016

14. Fahn S, Tolosa E, Marin C: Clinical rating scale for tremor, in Jankovic J, Tolosa E (eds): Parkinson's Disease and Movement Disorders. Baltimore: Urban \& Schwarzenberg, 1988, pp 225-234

15. Fang W, Chen H, Wang H, Zhang H, Puneet M, Liu M, et al: Essential tremor is associated with disruption of functional connectivity in the ventral intermediate Nucleus - motor cortex-cerebellum circuit. Hum Brain Mapp 37:165-178, 2016

16. Gironell A, Ribosa-Nogué R, Pagonabarraga J: Withdrawal of visual feedback in essential tremor. Parkinsonism Relat Disord 18:402-404, 2012

17. Glickstein M: How are visual areas of the brain connected to motor areas for the sensory guidance of movement? Trends Neurosci 23:613-617, 2000

18. Goldman MS, Ahlskog JE, Kelly PJ: The symptomatic and functional outcome of stereotactic thalamotomy for medically intractable essential tremor. J Neurosurg 76:924-928, 1992

19. Hua SE, Lenz FA, Zirh TA, Reich SG, Dougherty PM: Thalamic neuronal activity correlated with essential tremor. J Neurol Neurosurg Psychiatry 64:273-276, 1998

20. Ivanov B, Kaprelyan A, Bochev P, Dimitrov I, Grudkova M, Chaushev B, et al: (18F)-FDG PET/CT in essential tremor: preliminary result. J IMAB 21:914-921, 2015 
21. Joutsa J, Shih LC, Horn A, Reich MM, Wu O, Rost NS, et al: Identifying therapeutic targets from spontaneous beneficial brain lesions. Ann Neurol 84:153-157, 2018

22. Karahanoğlu FI, Van De Ville D: Dynamics of large-scale fMRI networks: deconstruct brain activity to build better models of brain function. Curr Opin Biomed Eng 3:28-36, 2017

23. Keogh J, Morrison S, Barrett R: Augmented visual feedback increases finger tremor during postural pointing. Exp Brain Res 159:467-477, 2004

24. Kondziolka D, Ong JG, Lee JY, Moore RY, Flickinger JC, Lunsford LD: Gamma Knife thalamotomy for essential tremor. J Neurosurg 108:111-117, 2008

25. Lipsman N, Schwartz ML, Huang Y, Lee L, Sankar T, Chapman M, et al: MR-guided focused ultrasound thalamotomy for essential tremor: a proof-of-concept study. Lancet Neurol 12:462-468, 2013

26. Liu X, Duyn JH: Time-varying functional network information extracted from brief instances of spontaneous brain activity. Proc Natl Acad Sci U S A 110:4392-4397, 2013

27. Llinás R, Yarom Y: Electrophysiology of mammalian inferior olivary neurones in vitro. Different types of voltage-dependent ionic conductances. J Physiol 315:549-567, 1981

28. Louis ED: Essential tremor. Lancet Neurol 4:100-110, 2005

29. Muthuraman M, Raethjen J, Koirala N, Anwar AR, Mideksa KG, Elble R, et al: Cerebello-cortical network fingerprints differ between essential, Parkinson's and mimicked tremors. Brain 141:1770-1781, 2018

30. Neely KA, Kurani AS, Shukla P, Planetta PJ, Wagle Shukla A, Goldman JG, et al: Functional brain activity relates to $0-3$ and 3-8 Hz force oscillations in essential tremor. Cereb Cortex 25:4191-4202, 2015

31. Preti MG, Bolton TA, Van De Ville D: The dynamic functional connectome: state-of-the-art and perspectives. Neuroimage 160:41-54, 2017

32. Schmidt R, Freidl W, Fazekas F, Reinhart B, Grieshofer P, Koch M, et al: The Mattis Dementia Rating Scale: normative data from 1,001 healthy volunteers. Neurology 44:964-966, 1994

33. Scobey RP, Johnson CA: Displacement thresholds for unidirectional and oscillatory movement. Vision Res 21:12971302,1981

34. Sharifi S, Nederveen AJ, Booij J, van Rootselaar AF: Neuroimaging essentials in essential tremor: a systematic review. Neuroimage Clin 5:217-231, 2014

35. Timmermann L, Gross J, Dirks M, Volkmann J, Freund HJ, Schnitzler A: The cerebral oscillatory network of parkinsonian resting tremor. Brain 126:199-212, 2003

36. Tröster AI, Pahwa R, Fields JA, Tanner CM, Lyons KE: Quality of Life in Essential Tremor Questionnaire (QUEST): development and initial validation. Parkinsonism Relat Disord 11:367-373, 2005

37. Tuleasca C, Najdenovska E, Régis J, Witjas T, Girard N, Champoudry J, et al: Clinical response to Vim's thalamic stereotactic radiosurgery for essential tremor is associated with distinctive functional connectivity patterns. Acta Neurochir (Wien) 160:611-624, 2018

38. Tuleasca C, Najdenovska E, Régis J, Witjas T, Girard N, Champoudry J, et al: Pretherapeutic motor thalamus restingstate functional connectivity with visual areas predicts tremor arrest after thalamotomy for essential tremor: tracing the cerebello-thalamo-visuo-motor network. World Neurosurg 117:e438-e449, 2018
39. Tuleasca C, Régis J, Levivier M: Essential tremor. N Engl J Med 379:595-596, 2018

40. Tuleasca C, Régis J, Najdenovska E, Witjas T, Girard N, Thiran JP, et al: Visually-sensitive networks in essential tremor: evidence from structural and functional imaging. Brain 141:e47, 2018

41. Tuleasca C, Witjas T, Najdenovska E, Verger A, Girard N, Champoudry J, et al: Assessing the clinical outcome of Vim radiosurgery with voxel-based morphometry: visual areas are linked with tremor arrest! Acta Neurochir (Wien) 159:2139-2144, 2017

42. Tuleasca C, Witjas T, Van de Ville D, Najdenovska E, Verger A, Girard N, et al: Right Brodmann area 18 predicts tremor arrest after Vim radiosurgery: a voxel-based morphometry study. Acta Neurochir (Wien) 160:603-609, 2018

43. Vasilakos K, Glass L, Beuter A: Interaction of tremor and magnification in a motor performance task with visual feedback. J Mot Behav 30:158-168, 1998

44. Witjas T, Carron R, Azulay JP, Regis J: Gammaknife thamamotomy for intractable tremors: clinical outcome and correlations with neuroimaging features, in MDS 17th International Congress of Parkinson's Disease and Movement Disorders. Milwaukee: International Parkinson and Movement Disorder Society, 2013, Vol 28, Abstract \#1247

45. Witjas T, Carron R, Krack P, Eusebio A, Vaugoyeau M, Hariz M, et al: A prospective single-blind study of Gamma Knife thalamotomy for tremor. Neurology 85:1562-1568, 2015

\section{Disclosures}

Prof. Girard is a consultant for Olea Medical. Prof. Régis is a consultant for Medtronic and Elekta. Prof. Thiran is a scientific advisor and member of the board of Intuitive Therapeutics SA.

\section{Author Contributions}

Conception and design: Tuleasca, Bolton, Van De Ville. Acquisition of data: Régis, Witjas, Girard. Analysis and interpretation of data: Tuleasca, Bolton, Van De Ville. Drafting the article: Tuleasca, Van De Ville. Critically revising the article: all authors. Reviewed submitted version of manuscript: all authors. Approved the final version of the manuscript on behalf of all authors:

Tuleasca. Statistical analysis: Tuleasca, Bolton, Régis, Najdenovska, Witjas, Girard, Faouzi, Van De Ville. Administrative/techni$\mathrm{cal} /$ material support: all authors. Study supervision: Van De Ville.

\section{Supplemental Information \\ Online-Only Content}

Supplemental material is available with the online version of the article.

Supplemenatary Data and Figs. 1 and 2. https://thejns.org/ doi/suppl/10.3171/2019.2.JNS183454.

\section{Correspondence}

Constantin Tuleasca: Centre Hospitalier Universitaire Vaudois, Lausanne, Switzerland. constantin.tuleasca@gmail.com. 